\title{
THEORETICAL TOOL MOVEMENT REQUIRED TO DIAMOND TURN AN OFF-AXIS PARABOLOID ON AXIS
}

\author{
D. C: Thompson
}

Decemer 19, 1975

\section{MASTER}

Prepared for U.S. Energy Research \& Development Administration under contract No. W. $7405-$ Eng- 46

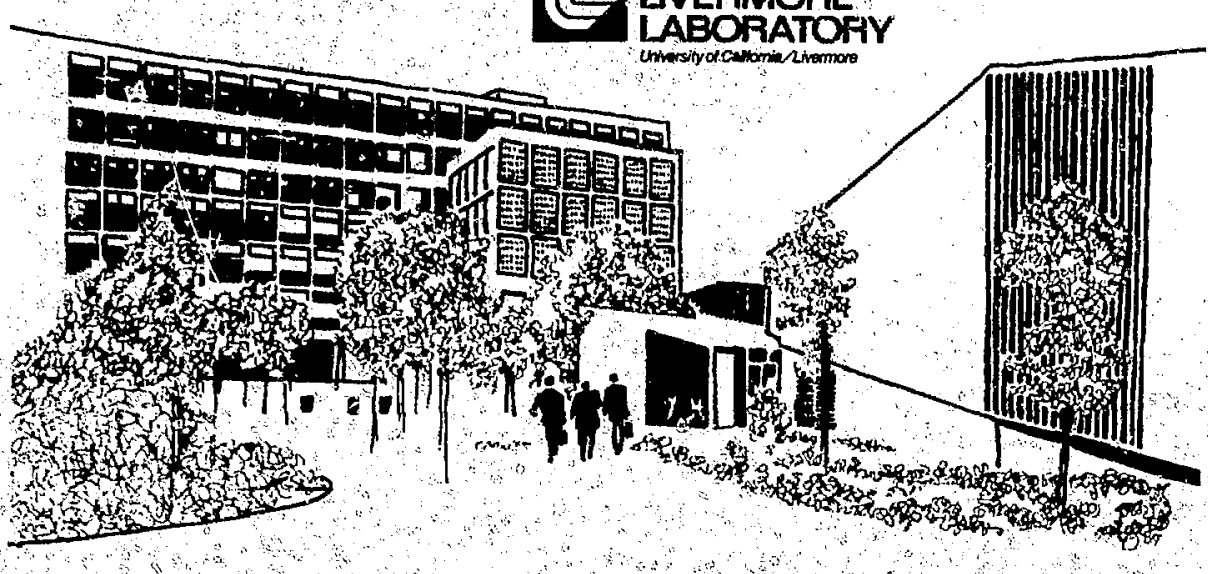

证

ing

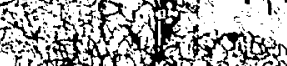

(c) 


\section{논 \\ LAMFENCE LNEFMOFE LABORATOFY

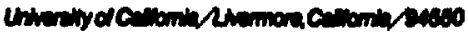

\section{VC1,-51984 \\ THEORETICAL TOOL MOVEMENT REQURED TO DIAMOND TURN AN OFF-AXIS PARABOLOID ON AXIS}

D. C. Thompon

HS. date: December 19, 1975

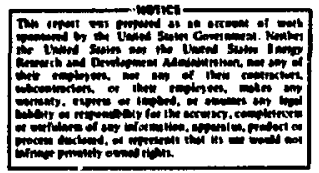


Contents

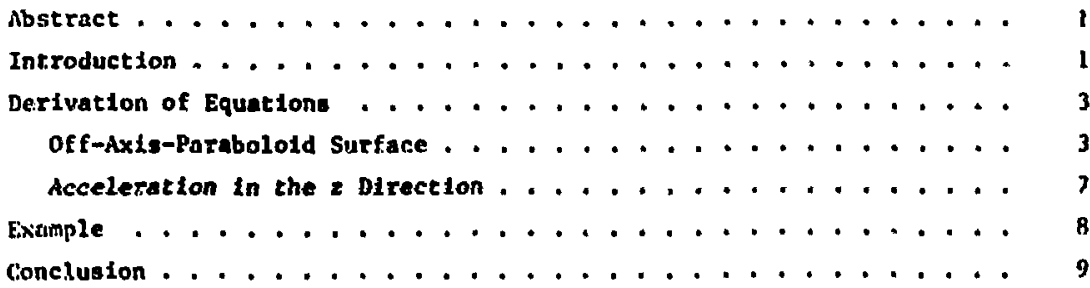

$-111-$ 


\section{THEORETICAI, TOOL MOVEMENT REQUIRED TO DIAMOND TURN AN OFF-AXIS PARABOL,OID ON AXIS}

\section{Abstract}

Current techniques for manufacturing off-axis parabololds are both expensive and Insufficiently accurate. An alternative method, turning the workpicce about Its axis on a diamond-turning machine, is presented, and the equat lons describlng the necessary tool movement are derived. A discusation of a particular case suggests that the proposed techrique is feasible.

\section{Introduction}

Iligh-quality, off-axis parabollc reflectors, required by the CTR and laser-fuston prograns at Lawrence Livermore Laboratory (LLL) and other ERDA laboratories, are currently manufactured by hand. There are several drawbacks to this method, Including lead times of up to year, costs in excess of $\$ 75,000$ for anall reflector, and unsatiafactory 1imits to the tolerances obtainable. Th1s situation has led to a search for cheaper and more accurate methods of manufacturing off-axis paraboloids.

One technique under consideration would involve mounting the workpiece at its normal position relative to the paraboloid's axis of rotation and cutting the reflector on a diamond-turning machine. Although this method should generate a reflector to the deutred tolerances, the size of the macinine required can be exceasive. The los Alamos Laboratory, for Inatance, is considertng the design of a So-in.-diameter, off-axis paraboloid, which would requiri a turning machine with a 20-ft swing. In addition, there is no guarantee that the size requireants "ill not continue to grow.

is an alternative to the technique outlined above, it has been suggested (by J. Bryan of LLl, and by members of the Prectsion Machining Development Group at Unton Carbide's Y-12 plant in Oak Ridge. Tennessee) that optical quality off-axis parabololda could be cut directly on a diamond-turning machine. The central axis of the workplece (the off-axis parabolotd) would be mounted concentric with the axis of rotation of the machine. A 
numerically controlled (NC) tool slide would vary the depth of cut in coordination with rotational position of the workpiece and radial distance from the axis of rotation (Fig. 1).

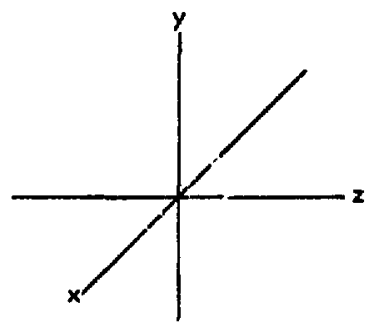

$\mathbf{z}$

From NC control to slide

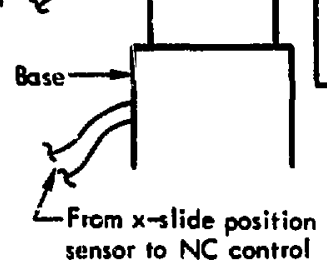

Slide

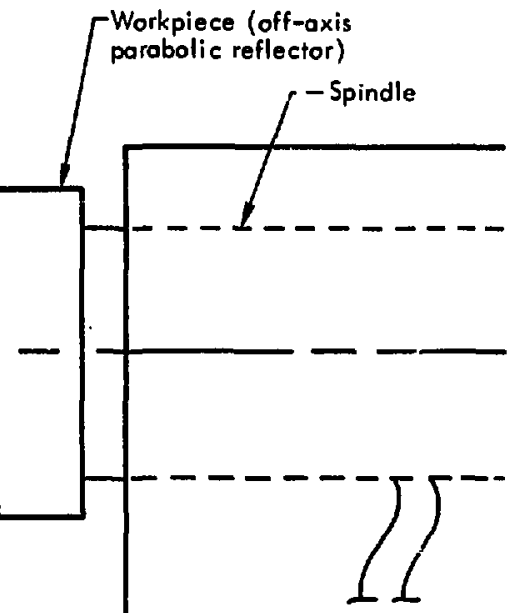

From spindle position sensor to NC control

Fig. 1. Conceptual diagram of the proposec mochining method. The base is numertcally controlied to move in the $x$ and $z$ directions. The alide gives an additional movement in the $z$ direction in coordination with the spindle position and the position of the tool in the $x$ direction.

Because of the "potato chip" shape of an iff-axis parabolic reflector there is a maxinum and minimum $z$ for any give radius. The difference, $\Delta z$, between $z_{\max }$ and $z_{\min } 18$ greatest at the oute, perimeter of the workpiece. The maximum displacement, $\Delta z_{\max }$, determines tie maximum distance traveled by the additional slide.

In the following discussion the equations necessary to implement such a Bystem are derived, and a particular case is analyzed. 


\title{
Derivation of Equations
}

\author{
OFF-AXIS-PARABOLOTU SURFACE
}

To derive an equation describing the surface of an off-axis paraboloid with respect to its zentral axis we first write the general equation for a parabolo1d:

$$
z=\frac{x^{2}+y^{2}}{c}
$$

where c is a constant. Figure 2 graphically displaya a parabolic raflector superimposed on an $x, y, z$ coordinate syatem.

The dusired equation can be obtained by performing two Iinear transforms on Eq. (1) - one translational and the other rotational. Figure 3 shows the axes translated to a new origin $0^{\prime}$ and rotated through $\theta$.

\section{Translation}

Assume that the point of origin of the transformed set of axes lies at a distance $s_{y}$ from the original point of origin along the original. $y$ axis. Further assume that there is no transformation along the original $x$ axis. The necessary trans-

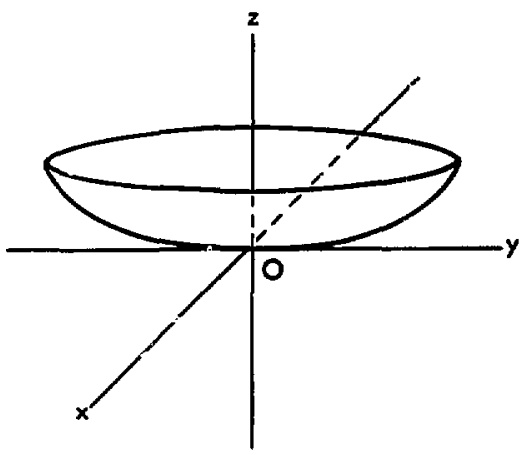

F18. 2. The relationship of the parabolic reflector to the coordinate axes.

formation in the 2 direction, then, is

$$
s_{z}=\frac{x^{2}+y^{2}}{c}=\frac{(0)^{2}+s_{y}^{2}}{c}=\frac{s_{y}^{2}}{c}
$$

Thus, 


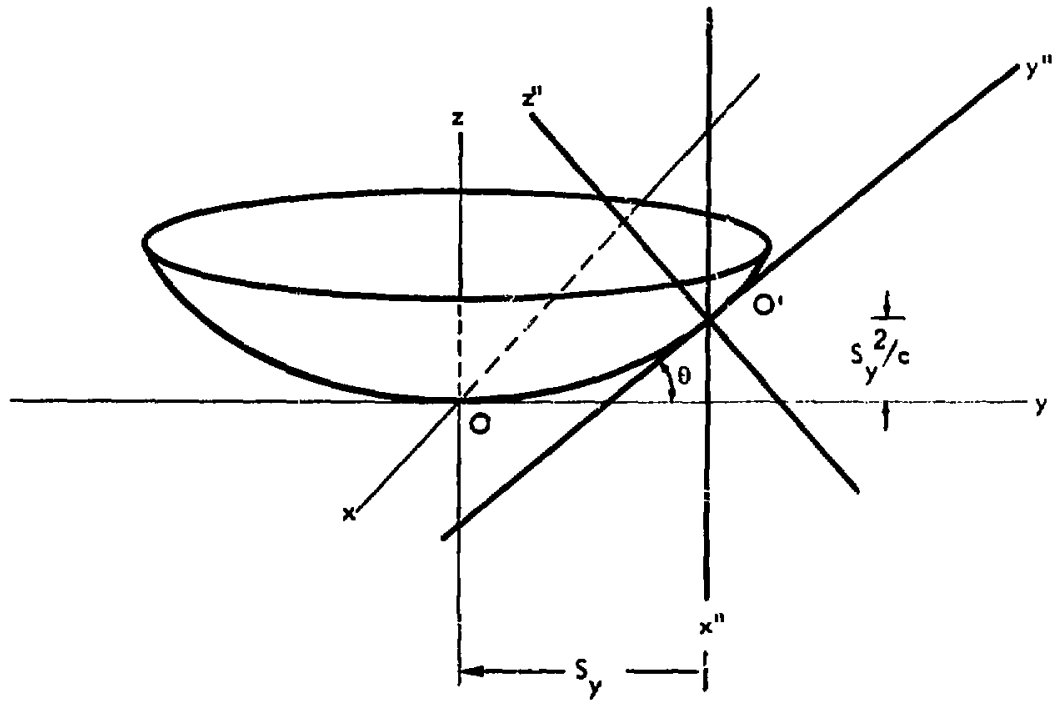

F18. 3. The coordinate axes translated to the new origin, $0^{\prime}$, and rotated through $\theta$ to make $z$ " perpendicular to the reflectior surface.

$$
\begin{aligned}
& x=x^{\prime}, \\
& y=y^{\prime}+s_{y}, \\
& z=z^{\prime}+\frac{s_{y}^{2}}{c},
\end{aligned}
$$

where $x^{\prime}, y^{\prime}$, and $z^{\prime}$ represent the transformed axp.s.

Using Eqa. (1) and (3), the equation describ:ig the surfiace may then be written as

$$
z^{\prime}+\frac{s_{y}^{2}}{c}=\frac{x^{\prime 2}+\left(y^{\prime}+s_{y}\right)^{2}}{c}
$$


which reduces to

$$
z^{\prime}=\frac{x^{, 2}+y^{, 2} \div 2 y^{\prime} s}{c} .
$$

\section{Rotation}

Next we must determine the angle $\theta$ through which the axes must be rotated to make the $z "$ axis normal to the surface at the new point of origin. We know that

$$
\operatorname{can} \theta \cdot \text { olope }=\frac{d z}{d y}
$$

and, from Eq. (1), that

$$
\frac{d z}{d y}=\frac{d}{d y}\left(\frac{x^{2}+y^{2}}{c}\right) \text {. }
$$

Since $x=0$ along the $y$-axis,

$$
\frac{d z}{d y}=\frac{2 y}{c}
$$

Translating to the new or $1_{\mathrm{B}}$ in, $0^{\prime}$, we get

$$
y=s_{y}
$$

and, from Eqs, (8) and (9),

$$
\frac{d z}{d y}=\frac{2 s_{y}}{c} \text {. }
$$

Using Eqs. (6) and (10; we get

$$
\tan \theta=\frac{2 s_{y}}{c} \text { : }
$$

and

$$
\theta=\arctan \frac{2 s_{y}}{c}
$$


For rotation around the $z^{\prime}$ axis,

$$
\begin{aligned}
& x^{\prime}=x^{\prime \prime}, \\
& y^{\prime}=y^{\prime \prime} \cos \theta-z^{\prime \prime} \sin \theta, \\
& z^{\prime}=z^{\prime \prime} \cos \theta+y^{\prime \prime} \sin \theta,
\end{aligned}
$$

where $\theta=\arctan 2 s_{y} / 2$.

Using Eqs. (5) and (13) we get

$$
\begin{aligned}
& z^{\prime \prime} \cos \theta+y^{\prime \prime} \sin \theta \\
& \quad=\frac{x^{\prime \prime 2}+\left(y^{\prime \prime} \cos \theta-z^{\prime \prime} \sin \theta\right)^{2}+25 y\left(y^{\prime \prime} \cos \theta-z^{\prime \prime} \sin \theta\right)}{c} .
\end{aligned}
$$

which reduces to the quadratic equation

$$
\begin{aligned}
0= & \left(\frac{4 s_{y}^{2} \cos \theta}{c^{3}}\right) z^{\prime \prime 2} \\
& \quad\left(1+\frac{4 s_{y}^{2}}{c^{2}}+\frac{4 s_{y}}{c^{2}} y^{\prime \prime} \cos \theta\right) z^{\prime \prime}+\left(\frac{y^{\prime 2} \cos 9}{c}+\frac{x^{\prime \prime 2}}{c \cos \theta}\right) .
\end{aligned}
$$

\section{Surface Equation}

Only the lower set of values of $z^{\prime \prime}$ is of interest. Thus, the solution set for the equation of the form

$$
0=a x^{\prime 2}+b x^{\prime \prime}+c
$$

will be described by the quadratic equation

$$
z^{\prime \prime}=\frac{-b-\left(b^{2}-4 a c\right)^{1 / 2}}{2 a} .
$$

Using Eqs. (15) and (17), the equation describing the surface of an off-axis paraboloid can be written as 


$$
z^{\prime \prime}-\frac{\left\{1+\frac{4 s^{2}}{c^{2}}+\frac{4 S_{y}}{c^{2}} y^{\prime \prime} \cos \theta\right\}-\left\{\frac{4 S_{y}}{c^{2}}\left[y^{\prime \prime}\left(\frac{c^{2}}{2 S_{y}}+2 S\right) \cos \theta+\frac{c^{2}}{2}+\frac{c^{4}}{16 s^{2}}+s_{y}^{2}-x^{\prime \prime 2}\right]^{1 / 2}\right\}}{\frac{8 s_{y}^{2}}{e^{3}} \cos \theta}
$$

In polar coordinates the solution may be written as

$$
=\frac{\left[1+\frac{4 s_{y}^{2}}{c^{2}}+\frac{4 s_{y}}{e^{2}}+\cos 0 \cos 0\right] \cdot\left\{\frac{4 s_{y}}{c^{2}}\left[\left(\frac{c^{2}}{2 s_{y}}+2 s_{y}\right) r \cos 0 \cos 0+\frac{c}{2}+\frac{c^{4}}{16 s^{2}}+s_{y}^{2}-z^{2}-1 n^{2} y\right]^{1 / 2}\right\}}{\frac{8 s_{y}^{2}}{e^{3}} \cos \theta} .
$$

where $y^{\prime \prime}=r \cos \phi, x^{\prime \prime}=r \ln \phi, r$ is the radius, and $\phi$ is the rotation about the $z$ axis with the $y^{+}$axis as the origin.

\section{ACCELERATION IN THE a DTRECTION}

The Instantaneous acceleration in the 2 direction with respsct to the ponttion $\phi$ may be obtained by taking the second derfvative of $z$ with respect to $\phi$ :

$$
\begin{aligned}
& \frac{d^{2} z}{d \phi^{2}}=\frac{c^{3}}{8 s_{y}^{2} \cos \theta}\left(\left[-\frac{4 S y}{c^{2}} r \cos \theta \cos \phi\right]-\frac{2 S y}{c^{2}}\left\{\left(\frac{c^{2}}{2 S_{y}}+2 S y\right) r \cos \theta \cos \phi\right.\right. \\
& \left.+\frac{c^{2}}{2}+\frac{c^{4}}{16 s_{y}^{2}}+s_{y}^{2}-r^{2} \sin ^{2} \phi\right]^{-1 / 2}\left[-\left(\frac{c^{2}}{2 S_{y}}+2 s_{y}\right) r \cos \theta \cos \phi\right. \\
& \left.-2 r^{2} \cos ^{2} \phi+2 r^{2} \sin ^{2} \phi\right]+\left[-\frac{1}{2}\right]\left[-\left(\frac{c^{2}}{2 s_{y}}+2 s_{y}\right) r \cos \theta \sin \phi\right. \\
& \left.-2 r^{2} \sin \phi \cos \phi\right]^{2}\left[\left(\frac{c^{2}}{2 s}+2 s\right) r \cos \theta \cos \phi+\frac{c^{2}}{2}\right. \\
& \left.\left.\left.+\frac{c^{4}}{16 s_{y}^{2}}+s_{y}^{2}-r^{2} \sin ^{2} \phi\right]^{-3 / 2}\right\}\right)
\end{aligned}
$$




\section{Example}

To determine whether or not cutting an off-axis parabolic reflector by the suggested method would be feasible, a particular situation was analyzed. The reflector used as an example is required by the LLI. CTR program and has the following parameters:

$$
\begin{aligned}
\text { Focal Jength } & =24.818 \mathrm{in} . \\
s_{y} & =23.972 \mathrm{in} . \\
c & =36.7887 \\
\text { Reflector radius, } r & =6.571 \mathrm{in} . \\
\theta & =52.50^{\circ}
\end{aligned}
$$

The displacement, $\Delta z_{\text {max }}$, determining the maximum travel required of the additional silde can be obtained from Eq. (18).

$$
\begin{aligned}
\Delta z_{\max } & =z_{\max }-z_{\text {min }} \\
& =0.723 \mathrm{In} .-0.240 \mathrm{In} . \\
& =0.483 \mathrm{In} .
\end{aligned}
$$

The maximum displacement required of the varlable position tool slide, then, would be less than $1 / 2 \mathrm{ln}$.

Using Eq. (20), the maximum acceleration, which also occurs at the outer perfmeter, is found to be

$$
\left(\frac{\mathrm{d}^{2} \mathrm{z}}{\mathrm{d \phi ^{2 }}}\right)_{\max }=0.977 \mathrm{1n} . / \mathrm{rad}^{2} .
$$

This figure may be converted to in. $/ a^{2}$ by multiplying by

$$
\left(2 \pi \frac{\mathrm{rad}}{\mathrm{rev}} \cdot \mathrm{rpm} \cdot \frac{1 \mathrm{mir} r}{60 \mathrm{~s}}\right)^{2}
$$

Thus, for example, the acceleration of the tool required for a cutting speed of $100 \mathrm{rpm}$ would be $107.14 \mathrm{In} . / \mathrm{s}^{2}$. This figure is roughly one-fourth of the value of the acceleration of gravity. 


\section{Conclusion}

We can conclude from the preceding analysis and discussion that $1 \mathrm{t}$ would be possible to turn off-axts parabololds on one of the LLL diamonci-turning machines. This method would permit satisfactory diamond-turning-machine tolerances and surface finlsh, would require a relacively small machine, and would take less time than the hand method.

To Implement this technique, several modificationg would have to be made to one of LLL's two diamond-turning machines. These would include the addition of a fast-response tool slide, a control system to be integrated with the existing NC unit, and position sensors on the slides and spindle. These modifications would give the dlamond-turning machine much greater versatility. The manufacture of complex shapes other than off-axis paraboloids would also be possible.

The cost of manufacturing off-axis parabololds by the proposed method will depend on the cost of the required modifications and the number of reflectors to be machined. However, because of the present high manufaccuring cost of and antlctpated increase In dentand for off-axis parabolic reflectors, It is probable that the manufacturing technique outlined here will become cost competitive.

$\mathrm{KC} / 1 \mathrm{t}$ 